\title{
Biosensors: A Comprehensive Review and Its Role in Cancer Treatment
}

\author{
Shashikant Patil \\ Member, IEEE
}

\author{
Chinmaya Vyas \\ SVKMs NMIMS, Mumbai
}

\begin{abstract}
The paper gives a brief outline regarding cancer and different types of cancers, their symptoms and the area of the body that is affected by them. The paper also gives an overview regarding biosensors their rudimentary characteristics and the abridgment between biosensor and cancer treatment. The different types of biosensors and their utility in different environments and conditions gives us cutting edge in terms of cancer treatment. Biosensors have indeed brought a revolution in the field of medical diagnosis and continue to affect the world with their unique characteristics and utility.
\end{abstract}

\section{INTRODUCTION TO CANCER}

Cancer is one of the prominent causes of deaths in the world (http://www.cancer.org.). Cancer which is actually a malignant tumour and can also is termed as a subset of neoplasm. When the cancer cells bifurcate uncontrollably they form lumps or heap of tissues which are termed as tumours. These lumps release hormones that inhibit the various body functions, as they interfere with the functioning of the several other parts of the body. The unceasing growth is the prominent factor behind the spreading of cancer. Normal cells in the body follow an orderly path of growth, division, and death. Cancerous cells do not respond to the chemical responses of the other cells. Benign Tumours are not life threatening since they do not invade other parts of the body. Two main types of genes play a role in development of cancer. Proto-oncogenes are naturally present in cells and help them to grow and divide. But a damaged Proto-oncogene may become an oncogene (a cancer gene). They direct cells to keep on growing and dividing, ignoring the normal stop signals. Suppressor genes are the second type that may be involved in cancer. These genes help to control cell growth and division. Damage to a suppressor gene can stop it from working, knocking out the cell's normal control over growth and division.

\section{SYMPTOMS OF CANCER}

It is not easy to identify a cancer. Its symptoms are quite varied and depend on where the cancer is located, where it has spread, and how big the tumour is. Some of these include a new lump on particular part of body, abnormal bleeding, a prolonged cough, unexplained weight loss, change in bowel movement. Few other cancers symptoms may not be physically visible

Table. Shows the various body parts affected by cancer and their symptoms.

\begin{tabular}{|l|l|}
\hline Cancer type/part of the body affected by cancer & Symptoms \\
\hline Melanoma(skin cancer) & Mole on a skin \\
\hline Oral cancers & White patches on skin \\
\hline Pancreas cancer & Yellowing of skin and eyes \\
\hline Colon cancers & Diarrhoea ,constipation and change in stool size \\
\hline Bladder cancers & Frequent or infrequent urination \\
\hline Brain cancer & Vertigo and headaches \\
\hline Lung cancer & Coughing and shortness of breath \\
\hline
\end{tabular}

Causes of Cancer

\begin{tabular}{|l|l|}
\hline Cause of cancer & Affected area \\
\hline Carcinogens & Damages DNA \\
\hline Human papillomavirus & Cervical Cancer \\
\hline hepatitis B and C & Liver cancer \\
\hline Epstein-Barr virus & Childhood Cancer \\
\hline Human papillomavirus & Genes of a cell \\
\hline
\end{tabular}




\section{New modalities helping the cancer world}

- Fiber optics microscope-This technique will help physician to detect cancerous diseases at early stage. It is helping clinicians target ideal location on lesion prior to and during surgical biopsies and by capturing high resolution images of tumour margin in real time.

- Advances in Radiology and molecular biology technology that may offer new possibility in cancer surveillances.

- Recent advancement includes detection of ovarian cancer by positron emission topography.

- For breast cancer, role of imaging techniques are used. Roles like mammography, ultrasonography, magnetic resource imaging, Positron emission tomography or mammography.

With the advancements being carried out in the field of cancer diagnosis several technologies have evolved over the years and biosensor technology is one of them. The detection of the biological analyte by converting it into an electrical signal is the technique through which biosensor aids in cancer detection. In the paper we would be discussing about biosensors and their application in the field of cancer diagnosis.

\section{Discussions AND Results}

\subsection{Biosensors and Cancer}

The main function of the biosensor being detection of biological analyte and the further processing of the electrical signal takes place for diagnosis purpose. Biosensor which is highly sensitive and specific result can be seen in the Biomarker technology but the more prominent techniques is Biosensor. The field of bio sensing involves sensing of specific elements from a complex mixture thus specificity is the key in the field. Optical biosensors which are far better than the other analytical techniques provide a suitable podium for the devices where specificity is a key factor. Thus optical biosensors are highly specific, reproducible, sensitive, rapid devices. Despite so much research in the field of biosensors the overall progress chart of the biosensors is accelerating at a very slow pace. The major obstacles to exploitation have been fundamentally related to the presence of biomaterial in the biosensor (immobilization of bio molecules on transducers, stability of enzymes, and antibodies), the development of the sensor device (sensitivity and reproducibility issues), and the integration of optical biosensors into complete systems [2]. Another major problem for the realistic mass production of biosensors has been the cost factor.

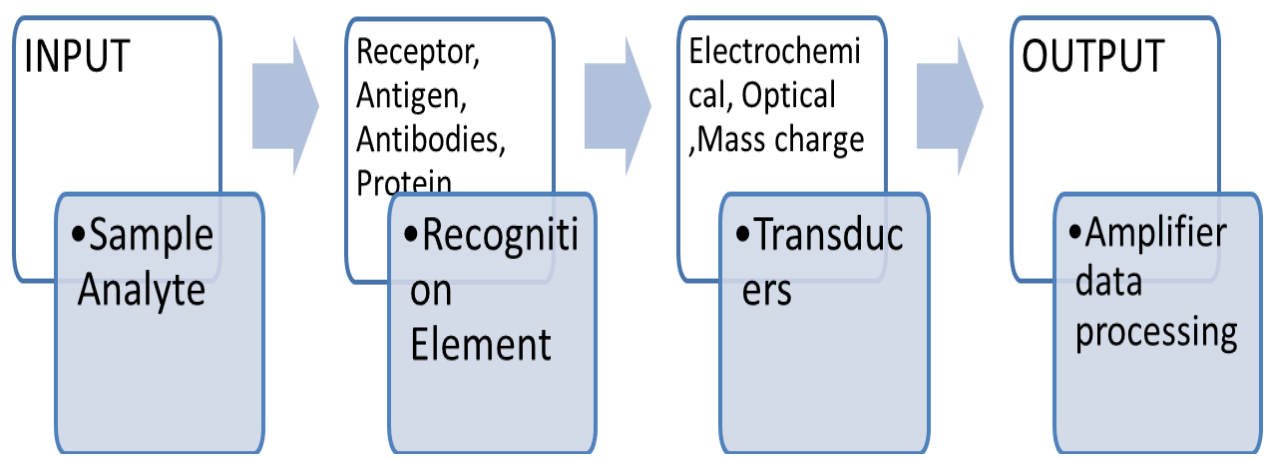

Figure1. The figure shows the detailed description of the working of the biosensor where from the sample analyte the specific element is recognised then the transducers convert the following physical property into the electrical signals which are then processed accordingly to procure desired results.

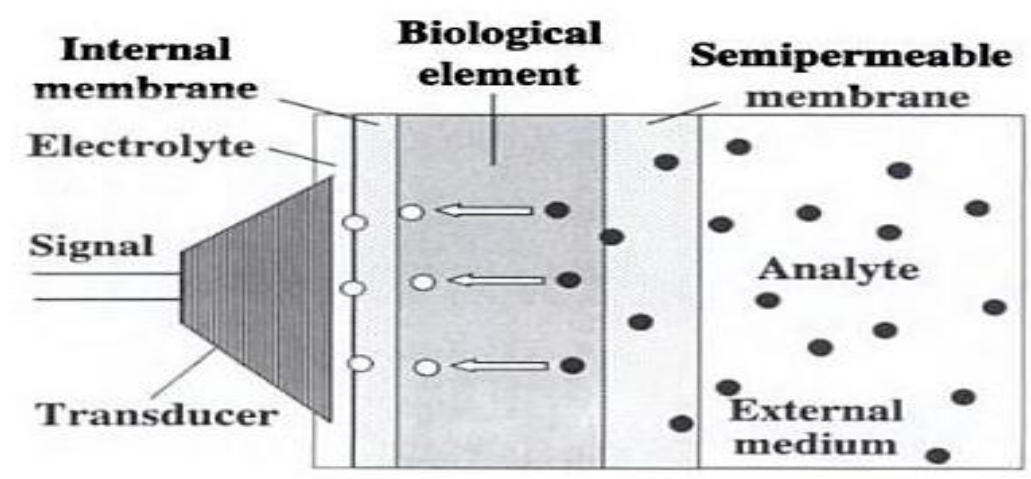

Figure2. Schematic of a generalised biosensor. 
In the figure above a target analyte (illustrated by solid circles) in the external medium must be able to enter the biosensor. The external membrane of biosensor must be permeable to the analyte, and if possible, exclude other chemical species that the biosensor might also be sensitive to. The biological element inside the biosensor then interacts with the analyte and responds in some manner that can be detected by a transducer [4]. The biological element may convert the analyte to another chemical species (represented by hollow circles) through a biochemical reaction, produce or release another chemical product in response to the analyte stimulus; change its optical, electrical or mechanical properties; or make some other response that can be reliably quantified. There may be another internal membrane near the transducer which might have different permeability properties than the external membrane. The output signal from a biosensor depends on the type of transducer it uses. The transducer may be a conventional electro-chemical sensor, or may be based on another technology.

Lung cancer shows the highest mortality rate worldwide (1.3 million/year) with respect to other common cancers as prostate, breast and colon cancer. It is the second most common cancer in men and the third in women (22\% of all cancer cases). The cancer also shows variations in incidence rate depending upon geographical location of the world, for instance, the highest rates of the diseases in men are determined in Europe, particularly eastern and central Europe, and northern America early diagnosis for this disease. Among European countries, Hungary and Poland have the highest male rates whereas Sweden and Malta have the lowest, respectively (Fig. 1) [1, 2]. The treatment of the disease is long and difficult with survival scarcely attains to 5 years [5]. The most critical point for best prognosis is to diagnose the disease at its early stage. For this aim, divers' methods have been used including chest X-ray, computerized tomography, magnetic resonance imaging, positron emission tomography, sputum cytology and biopsy. Although some of these methods are not suitable for all people due to the other pathologies that the patient may have. Also since the current diagnostic tools are also expensive and time consuming, hence, a new sensitive and rapid method for screening is necessary for lung cancer Estimated scenario of cancer in various geographical location in world. The treatment for cancer is long going procedure and difficult and the survival scarcely attains to be 5year,best solution is detection in early stage and for this aim there are many method like chest x-ray, computerized tomography, biopsy, imaging techniques and so on but some of these method is not suitable because of the pathologies that patient may have.(National cancer Institute). There are various technology but the most effective and promising way for diagnosis of cancer is SENSOR, which is rapid, easy applications and sensitive. Getting high specific and sensitive result by this technology for the detection of biomarker from blood sample may provide easy and early detection of cancer without any pain and with non-invasive techniques. Tumour markers are substances found in the blood, urine, stool, other bodily fluids, or tissues of some patients with cancer. It may be used to help diagnose cancer, predict a patient's response to certain cancer therapies, check a patient's response to treatment, or determine whether cancer has returned [6]. The commonest cancer types include breast, lung, prostate and colon carcinomas; however, the rate of mortality is quite low for breast and prostate cancers when compared with lung cancer. Moreover, the mortality and incidence rates of lung cancer show similarity and this increases the importance of a number of tumour markers are currently being used for a wide range of cancer types. Although most of these can be tested in laboratories that meet standards set by the Clinical Laboratory Improvement Amendments, some cannot be and may therefore be considered experimental.

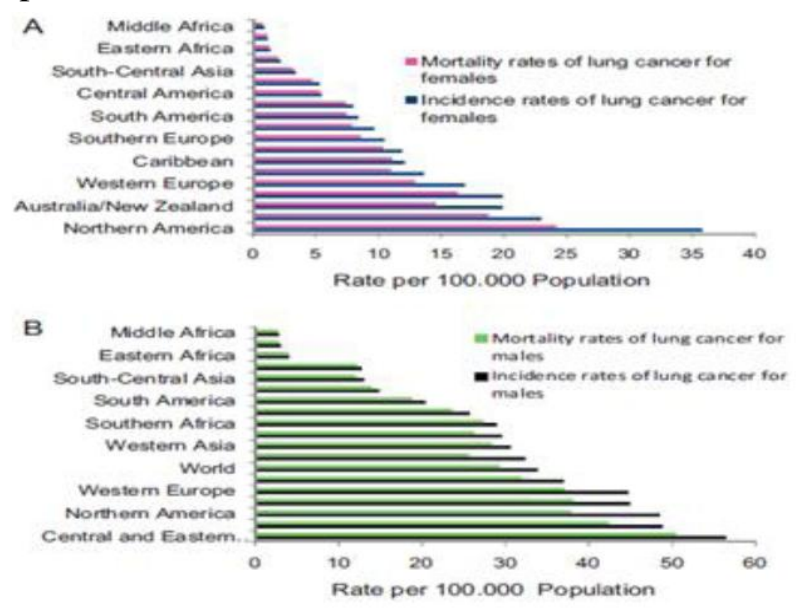

Figure3. Mortality and incidence rates of lung cancer at different locations: (A) Females (B)Males. 
Some tumour markers that screening method are listed below-

\begin{tabular}{|c|c|c|c|}
\hline Tumour markers & Cancer type & Tissue analysed & Advantages \\
\hline ALK gene rearrangements & $\begin{array}{l}\text { Lung cancer \& } \\
\text { lymphoma }\end{array}$ & Tumour & $\begin{array}{l}\text { To help determine treatment } \\
\text { and prognosis }\end{array}$ \\
\hline Alpha-fetoprotein (AFP) & $\begin{array}{l}\text { Liver cancer and germ } \\
\text { cell tumours }\end{array}$ & Blood & $\begin{array}{c}\text { To help diagnose liver } \\
\text { cancer and follow response } \\
\text { to treatment; to assess stage, } \\
\text { prognosis, and response to } \\
\text { treatment of germ cell } \\
\text { tumours }\end{array}$ \\
\hline $\begin{array}{l}\text { Beta-2-microglobulin } \\
\text { (B2M) }\end{array}$ & $\begin{array}{l}\text { Multiple myeloma, } \\
\text { chronic lymphocytic } \\
\text { leukaemia, and some } \\
\text { lymphomas }\end{array}$ & $\begin{array}{l}\text { Blood, urine, or } \\
\text { cerebrospinal fluid }\end{array}$ & $\begin{array}{l}\text { To determine prognosis and } \\
\text { follow response to } \\
\text { treatment. }\end{array}$ \\
\hline $\begin{array}{c}\text { Beta-huma chorionic } \\
\text { gonadotropin (Beta-hCG) }\end{array}$ & $\begin{array}{l}\text { Choriocarcinoma and } \\
\text { testicular cancer }\end{array}$ & Urine or blood & $\begin{array}{l}\text { To assess stage, prognosis, } \\
\text { and response to treatment. }\end{array}$ \\
\hline $\begin{array}{c}\text { Chromosomes } 3,7,17 \text {, and } \\
9 \text { p21 }\end{array}$ & Bladder cancer & Urine & $\begin{array}{l}\text { To help in monitoring for } \\
\text { tumour recurrence }\end{array}$ \\
\hline Cytokeratin fragments $21-1$ & Lung cancer & Blood & $\begin{array}{l}\text { To help in monitoring for } \\
\text { recurrence }\end{array}$ \\
\hline
\end{tabular}

\section{LimitATIONS OF TUMOUR MARKERS}

Tumour markers are not full proof. Usually doctors need results from other tests, such as an x-ray or biopsy, to determine whether the presence of a particular tumour marker is a cause for concern. This is because:

1) An elevated tumour marker level may be caused by a condition or disease other than cancer.

2) Some tumour marker levels may be high in people without cancer.

3) Tumour marker levels may vary over time which clearly affects the consistency of the system. The level of a tumour marker may not rise until a person's cancer is advanced, which is not helpful for early detection, screening, or monitoring for recurrence [7].Now with the much of the development done in the field of biosensors selection of the specific biosensor in itself is a challenge and to simply the problem the biosensors are classified in a systematic manner for their selection:

\section{ON THE BASIS OF BIOLOGICAL ELEMENT}

a) Enzyme Biosensor: Enzymes because of its high selectivity and activity towards substrate are the best candidate to be used as biologically active materials. Most of the enzymes used in biosensor are Oxidizers but there are certain limitations as their activity is susceptible to $\mathrm{pH}$, temperature, ionic strength etc.

b) Microbial Biosensor: it uses microorganism as biological element .The metabolism of microorganism is used as basis of their activity. They are cheaper \& versatile as compared to other sensor elements.

c) Antibody Based Biosensor: It uses antibodies as sensor. They are immobilized on the surfaces by covalent /Ionic/ Vander wall attachment in conjugation with amino-carboxyl groups etc [8].

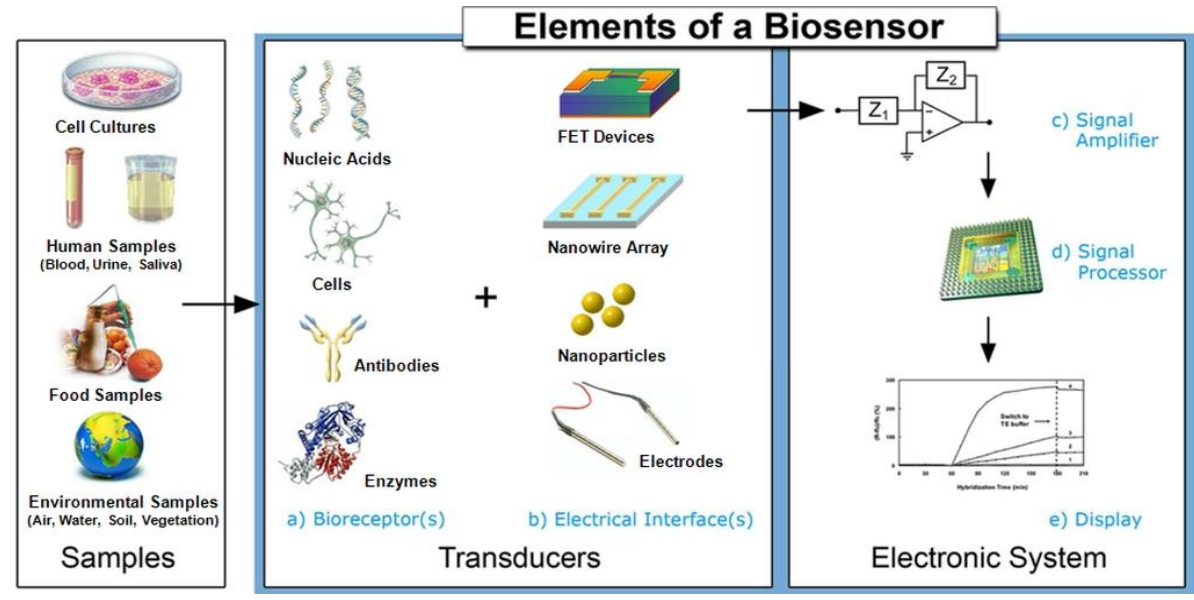

Figure4. Schematic of a typical biosensor [9] 


\section{ON THE BASIS OF TRANSDUCING ELEMENT}

\subsection{Transducers of Biosensors}

A transducer is a device that converts recognition signal events into electrical signals, which could be electrochemical (amperometric, potentiometry, conductimetry/impedimentary), optical (colorimetric, fluorescence, luminescence, interferometry), and mass change (piezoelectric/acoustic wave), and so on. The transducer is required to offer high throughput, high signal-to-noise ratio, relatively low instrumentation costs, good resolution and reproducible results. The sensitivity and discrimination ability of a differential sensor are critically dependent on the number of sensing elements. Types of Biosensor are

\subsection{Electrochemical Transducer}

They have been miniaturized to small pocket size devices. For example, the glucose biosensor is applicable for home use, realizing rapid point-of-care measurement. Amperometric and potentiometric transducers are the most commonly used among all the electrochemical transducers. Amperometric biosensors could monitor the current associated with the reduction or oxidation of electro activity. The potentiometric devices convert the bio recognition process into a potential signal in connection to the use of ion-selective electrodes (ISE). In recent years, more attention has been devoted to impedance transducers with the capacity of label-free detection. Electrochemical detection of proteins is also widely used. There are two main detection principles. The antibody-antigen interaction could cause changes in the electrochemical properties (conductivity or capacitance) of the sensor surface. In the ELISA based assays, the reporter antibody or antigen is labelled with enzymes such as horseradish peroxidase (HRP), or alkaline phosphatase (AP), which will catalyse substrates to produce an electro active species.

\subsection{Mass Sensitive Transducer}

Many physical and chemical processes are associated with mass changes. The piezoelectric quartz crystal resonator makes the microbalance possible. The application of an external electrical potential to a piezoelectric material produces internal mechanical stress. The QCM is a piezoelectric sensor comprises a thin quartz crystal disk coated with gold electrode. An oscillating electric field is applied across the device, inducing an acoustic wave that propagates through the crystal and meets minimum impedance when the thickness of the device is a multiple of the half wavelength of the acoustic wave, so the QCM device is accurately a thickness shear mode resonator. The mass of a thin layer attached to the surface of a crystal can be calculated from a measured change in the resonant frequency of the device. The resonance frequency has been proved to decrease linearly with increasing mass on the QCM electrode at a Nano gram level. QCM is a very sensitive mass-measuring device, and has been employed to observe real time biological events.

\subsection{Optical Transducers}

\section{1) $S P R$}

Optical transducers used in biosensors include fluorescence, interferometry and spectroscopy of optical waveguides, and surface Plasmon's resonance (SPR). SPR is one of the most common labelfree optical techniques used for bimolecular interaction detection. SPR biosensor is based on wavelength modulation and the Kretschmann geometry of the attenuated total reflection method. Briefly, a collimated polychromatic light beam from a halogen lamp passes through an optical prism and contacts a thin gold layer at a defined angle of incidence. Upon the incidence of the thin gold film, each light beam excited a surface Plasmon at a certain wavelength. The reflected light is collected by an instrument with measured channels. Acquired spectra were analysed in real time. The refractive index creates a characteristic dip in the detected light spectrum at a specific wavelength. Changes in refractive index cause this characteristic dip to shift. During the experiments a constant temperature is maintained to eliminate effects from temperature changes, so the changes are associated with the binding events occurring on the surface only, which could be quantified by tracking these changes in dip position. PR biosensors are sensitive to any refractive index change of the solution injected on the sensor surface. Refractive index mismatch of the buffer, the sample, as well as the running buffer can become a problem. Using a control serum solution that does not contain elevated levels of the antigen as running buffer is theoretically possible, but not feasible due to cost and health issues, while performing a sandwich assay instead of a direct assay will help in eliminating this problem 


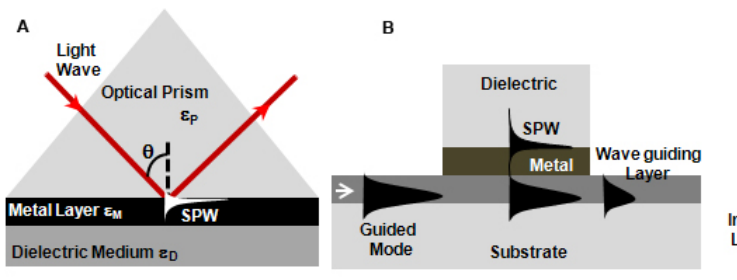

C
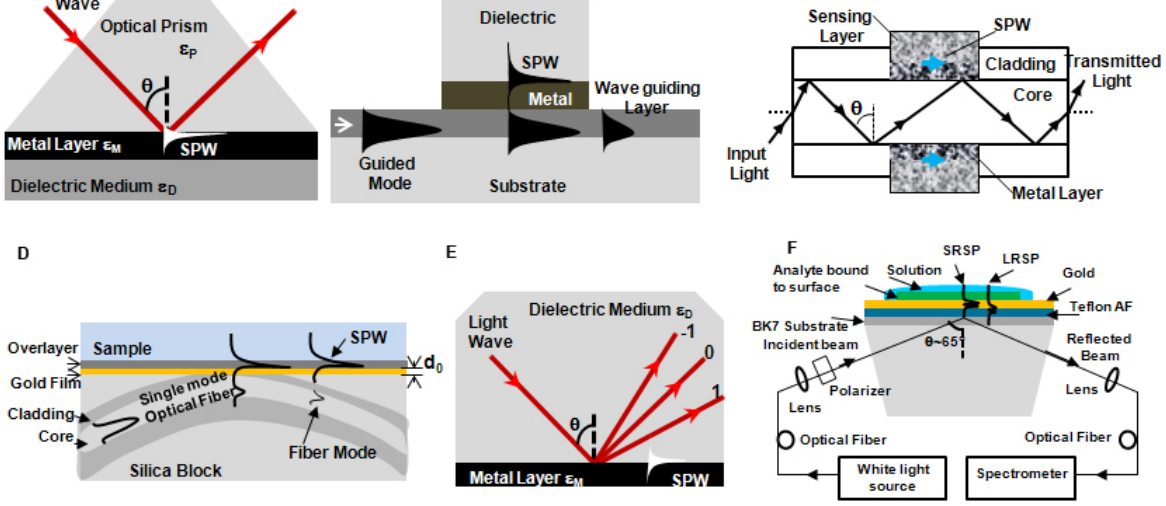

Figure5. Various SPR sensor configurations.(A) Prism coupling, (B) waveguide coupling, (C) optical fiber coupling, (D)side polished fibre coupling, $(E)$ grating coupling and $(F)$ long-range and short-range surface Plasmon (LRSP and SRSP)[10].

\section{2) SPR Imaging (SPRi)}

Recently, the SPR imaging (SPRi) technique has been at the forefront of optical label-free and realtime detection. SPRi systems are generally based on intensity modulation, measuring the reflectivity of monochromatic incident p-polarized light at a fixed angle. S-polarized light could also be measured, but is only used as reference signal to improve the image contrast and to eliminate artefacts. The sensing surface of the prism is coated with a thin gold or silver layer. The resonance conditions depend on the characteristics of the prism, metal and dielectric medium. The device can be used as a multichannel sensor when the sensor surface is divided into multiple sensing spots. Sprig could monitored hundreds of biological interactions continuously and simultaneously, and controlling the quality of the spotted array by selecting the measurement regions according to shape, size and quality.

\section{3) Fluorescence Biosensor}

\section{Chromophore}

Fluorescence techniques are very well suited to realizing the sensitive, reliable and reproducible detection of early cancer biomarkers, while fluorescence methods encompass several unique experimental parameters, for instance, excitation and emission wavelength, intensity, fluorescence lifetime and emission anisotropy, which are determined by the chromophore. A variety of Chromophore could be used, such as organic dyes, metal-ligand complexes, lanthanide chelates, and Nano crystals. Organic dyes are widely used for in vitro fluorescence quantification applications, for example, ELISA and real-time quantitative RT-PCR. The conjugation of organic dyes to bio molecules can be easily performed without significantly altering the biological functions. However, the spectral overlap problems of organic dyes need to be overcome for multiplexed approaches. Also, the fluorescence lifetime of organic dyes is too short for efficient discrimination.

\section{QDs as Chromophore}

Luminescent semiconductor Nano crystals were utilized as fluorescent biological labels for the first time in 1998. Semiconductor quantum dots that covalently coupled to biomolecules, could recognize specific antibodies or antigens as fluorescent probes in biological staining and diagnostics. The QDs that are synthesized in the organic phase possess better quality, but require additional post-treatment to gain water dispersibility. In contrast, QDs prepared in the aqueous phase possess excellent aqueous dispersibility, while fluorescence quantum yields are lower than these synthesized in the organic phase. Highly luminescent QDs have been readily achieved in the aqueous phase through several strategies.

\section{Micro fluidic Chip}

In static solid/liquid interface reaction systems, QDs are less active than organic dyes because the QDs are prone to deposit on the solid surfaces. To overcome the problem, micro fluidic chips based on the manipulation of a continuous liquid flow through micro fabricated channels are applied, and we found that QDs were compatible with micro fluidic chips. The ambulatory fluid accelerates the 
reaction of QD probes and targets, and prevents QDs from nonspecific deposition. These chips are highly sensitive for use in cancer biomarker assays. Our group developed a micro fluidic protein chip with QDs as fluorescent signal amplifiers for ultrasensitive and multiplexed assay of cancer biomarkers, and it was selective enough to be used in sera directly.

\section{Fluorescence Resonance Energy Transfer (FRET)}

FRET, which has been reviewed previously is a distance dependent energy transfer between a donor and an acceptor separated by a distance of 1-20 nm. As many bio molecular interactions occur in this distance range, FRET is frequently used for the parallel detection of several molecular interactions. The acceptor absorbs energy at the emission wavelength of the donor, but does not necessarily remit the energy fluorescently itself. The distance between the donor and acceptor molecules would affect the rate of energy transfer violently, making FRET very appealing for bio analysis because of its intrinsic sensitivity to D/A distance.

\section{BIOSENSOR CHARACTERISTICS}

The optimum design of electrochemical, optical, and other types of biosensor is detected by several basic physical properties of the measuring system, as well as those of the media in which measurement is made. Some of the most pertinent properties and characteristic behaviour of ideal biosensor are listed as follows:-

\section{Sensitivity}

It is usually defined as final steady state change in the magnitude of the biosensor output signal with respect to the change in concentration of a specific chemical species. There are many factors that determine the effective sensitivity of a given biosensor design to a target analyte. These include the physical size of the sensor, the thickness of the membranes and resulting mass transport of chemical species from the sample to the sensing region, and various processes that deactivate the biosensor or otherwise impair its operation over time. Ideally, the sensitivity of a given biosensor should remain constant during its lifetime and should sufficiently high to allow convenient measurement of the transducer output with electronic instrumentation.

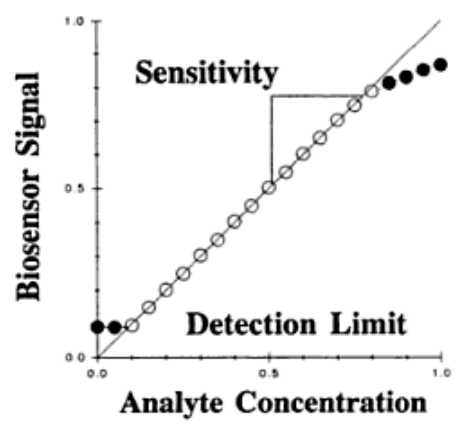

\section{Linearity}

A perfectly linear biosensor will have a constant sensitivity over the concentration range from zero to the maximum substrate concentration that can be physically dissolved in the measurement medium.If each biosensor has a unique, non-linear calibration curve, it still may be practically useful, but will require more complicated signal analysis to extract the desired information.

\section{Limit of detection}

Ideally, the lowest concentration of substrate that can be detected by a biosensor (as shown in figure) should be limited only by the resolution of the electronic instrumentation used for the measurement. In practical terms, other considerations cause the lower limit of detection to be higher.

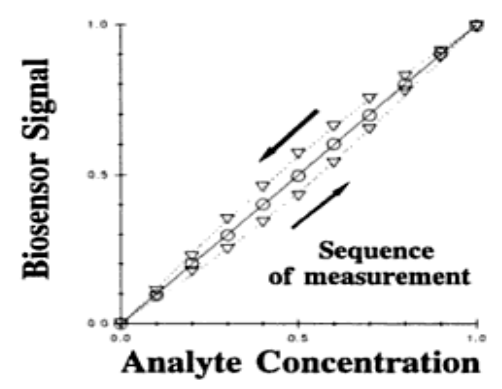


4. Hysteresis

An ideal biosensor should not be affected by its past history of measurements, and would have zero hysteresis. Hysteresis can also affect the transient responses of the biosensor. In some cases, hysteresis can be minimized by making small changes.

\section{Selectivity (Interference)}

The ideal biosensor will only respond to changes in concentration of the target analyte, and will not be influenced by the presence of other chemical species. Otherwise, false readings for the analyte concentration would be obtained if it is actually the concentration of the interfering species that is changing.

\section{Temperature Dependence}

All of the physical properties, such as diffusion coefficients, gas and chemical solubility's of the liquids, and solid materials used in the construction of a biosensor will vary with temperature. The rates of enzyme catalysed reactions are very temperature dependent. Heat can be produced in some chemical reactions, which can change the biosensor temperature.

\section{Signal to noise}

When the current is measured, the major source is Johnson noise due to random electron movements in the feedback circuit. The root mean square error for Johnson noise is given by,

$$
E_{n}=\sqrt{4 k R_{f} T f_{0}}
$$

Where $\mathrm{k}$ is Boltzmann's constant, $\mathrm{R}(\mathrm{f})$ is the amplifier feedback resistance, $\mathrm{T}$ is temperature in Kelvin and $\mathrm{f} 0$ is cut-off frequency of the amplifier.

Hence, the signal to noise ratio can be written as

$$
\frac{S}{N}=I \sqrt{\frac{R_{f}}{4 k T f_{0}}}
$$

The S/N ratio can be reduced by reducing the cut-off frequency of the amplifier, or by passing the output through analog filters. Another technique for improving the $\mathrm{S} / \mathrm{N}$ ratio of repeated signals is simple ensemble averaging. The averaging can be synchronized with the repetitive stimulus for this method.

\section{Lifetime}

An important property of biosensor is the length of time that they remain sensitive under normal conditions. The lifetime may be dependent on the total number of measurements made, or may depend on the magnitude of the analyte concentration measure. Higher concentrations may lead to more rapid losses in sensitivity. Another important property is the length of time that the biosensor can be stored between usages.

\section{Conclusion}

Biosensors have brought a revolution in the medical industry through there multiple uses and their properties which aid in cancer treatment.

The various characteristics and techniques have been discussed in the paper.

Biosensors can measure non polar molecules that do not respond to most measurement devices. They are as specific as the immobilized system used in them. They allow rapid continuous control. Heat sterilization is not possible as this would denature the biological part of the biosensor. The membrane that separates the reactor media from the immobilized cells of the sensor can become fouled by deposits. The cells in the biosensor can become intoxicated by other molecules that are capable of diffusing through the membrane. Changes in the reactor broth (i.e., $\mathrm{pH}$ ) can put chemical and mechanical stress on the biosensor that might eventually impair it. 
Thus for a biosensor the sensitivity should remain constant a small amount of deviation is tolerable but larger ones may cause error in the transducer output measurement. Biosensor should show a linear characteristics so that the signal(output) analysis is not tedious otherwise if a biosensor has a unique, non-linear calibration curve, it still may be practically useful, but will require more complicated signal analysis to extract the desired information. The biosensor should have a zero hysteresis and hence the pervious values of measurement should not affect the present ones. Biosensor should be selective in nature and the temperature characteristics should be constant not much variation should be there which afterwards affects the output. Another important aspect of the biosensor being the signal to noise ratio should be high and thus there shall be no interference of noise in the signal to be analysed further. Thus biosensor with their characteristics and utility continue of give new dimensions to medical science and health industry.

\section{Acknowledgement}

We have taken efforts in this research work. However, it would not have been possible without the kind support and help of many individuals and organizations. We would like to extend our sincere thanks to all of them. We would like to express our sincere thanks to industry persons and fellow colleagues for their kind attention and time.

\section{REFERENCES}

[1] Keith E. Herold and Avraham Rasooly, Biosensors and Molecular Technologies for Cancer Diagnostics, Taylor \& Francis 2012,Pages 3-40.

[2] By HuangxianJu, Zhang Xueji, Joseph Wang, NanoBiosensing: Principles, Development and Application

[3] N. Laxmi, A REVOLUTION IN THE DEVELOPMENT OF OPTICAL BIOSENSORS, International Journal of Pharmacy \& Technology, ISSN: 0975-766X CODEN: IJPTFI

[4] SundaramGunasekaran, Nondestructive Food Evaluation: Techniques to Analyze Properties and Quality

[5] www.cancer.org/acs/groups/content/.../documents/document/acspc-044552.pdf

[6] Patrick W. Nee, The Key Facts on Cancer: Everything You Need to Know About Cancer

[7] www.cancer.net/printpdf/24730

[8] SarveshRustagi and Pravesh Kumar, Biosensor and It's Application in Food Industry, Advances in Bioresearch Adv. Biores., Vol4 (2) June 2013: 168- 170

[9] DorotheeGrieshaber;, Robert MacKenzie;, Janos V"or"os and Erik Reimhult; Sensors 2008, 8, 1400-1458 ISSN 142482202008

[10] Xudong Fan, Ian M. White, Siyka I. Shopova, Hongying Zhu, Jonathan D. Suter, YuzeSunSensitive optical biosensors for unlabeled targets: A review. Analytica chimica acta 620(2008) 8-26

[11] Alexander P. Demchenko, Introduction to Fluorescence Sensing ISBN: 978-3-319-20779-7 (Print) 978-3-319-20780-3 (Online)

[12] YildizUludag and Ibtisam E. Tothill, Cancer Biomarker Detection in Serum Samples Using Surface Plasmon Resonance and Quartz Crystal Microbalance Sensors with Nanoparticle Signal Amplification, Anal. Chem., 2012, 84 (14), pp 5898-5904 DOI: 10.1021/ac300278p

[13] Calvert Louden, Ruth A. Roberts, Validating In Vitro Toxicity Biomarkers Against Clinical Endpoints Biomarkers in Drug Development: Practice, Application, and Strategy, DOI: 10.1002/9780470571224.pse273

[14] E Scott Sills, Screening the Single Euploid Embryo: Molecular Genetics in Reproductive Medicine, ISBN: 978-3-319-16891-3 (Print) 978-3-319-16892-0 (Online)

[15] Mei Hu ,Juan Yan, Yao He, Haoting Lu, LixingWeng, Shiping Song, Chunhai Fan and Lianhui Wang, Ultrasensitive, Multiplexed Detection of Cancer Biomarkers Directly in Serum by Using a Quantum Dot-Based Microfluidic Protein Chip, ACS Nano, 2010, 4 (1), pp 488-494

DOI: $10.1021 / \mathrm{nn} 901404 \mathrm{~h}$

[16] Vanessa Almendro, Andriy Marusyk, and Kornelia Polyak,Cellular Heterogeneity and Molecular Evolution in Cancer Annual Review of Pathology: Mechanisms of Disease, Vol. 8: 277-302, DOI: 10.1146/annurev-pathol-020712-163923 
[17] Kin-yaTomizaki Dr., Kenji Usui and HisakazuMihara,. Review: Protein-Detecting Microarrays: Current Accomplishments and Requirements, DOI: 10.1002/cbic.200400232

[18] Elfriede Simon, Biological and chemical sensors for cancer diagnosis, Measurement Science and Technology, Volume 21, Number 11

[19] Urs A. Meyer, Ulrich M. Zanger, and Matthias Schwab, .Omics and Drug Response Annual Review of Pharmacology and Toxicology Vol. 53: 475-502 (Volume publication date January 2013) DOI: 10.1146/annurev-pharmtox-010510-100502

[20] Se-Kwon Kim, Handbook of Anticancer Drugs from Marine Origin, ISBN: 978-3-319-07144-2 (Print) 978-3-319-07145-9 (Online).

\section{AUTHORS' BIOGRAPHY}

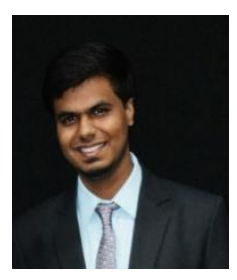

Chinmaya Vyas, is currently pursuing B. Tech in Electronics and Telecommunication Engineering from the SVKM'S NMIMS, shirpur, India. His research interest includes embedded system and robotics, antenna and radio frequency, sensors and control systems. He has published 04 articles in Conferences and International Journals.

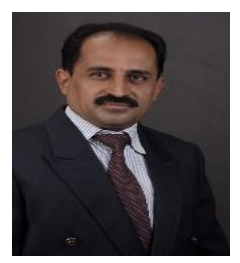

Prof. Shashikant Patil, is working as an Associate Professor in Electronics and Telecom Department in SVKM'S NMIMS, Shirpur, India. He is currently serving as an Associate Editor, Editorial Board Member, Technical Advisory Board Member, Potential Peer Reviewer and Journal Referee on at least 50 Journals. He is also having an association with Elsevier Editorial Series, Taylor and Francis, Springer Link Journals as a potential Peer Reviewer and Journal Referee. He is also associated with IEEE, ACM, CSI, ISTE, NHRDN, E4C and IETE as Professional member and nominated as an affiliate member on various committees of IEEE SPS and ComSoc. He is recipient of Best Researcher Award 2014 of SVKMs NMIMS Shirpur. He has published around 31 articles in various conferences and journals at National and International level. This year he has been selected as Regional Lead Ambassador for region 10 for IEEE Day 2015 and 2016 event. He is member IEEE RFID Technical Council and SIG Member of IoT. His research interests are Signal Processing and Imaging. He is also serving as an Editor in Chief for IEEE CRFID Newsletter. 